\title{
Scheduling problems with energy consideration: State of the art and opportunities
}

\author{
Ghita Lebbarl, Ikram El Abbassi ${ }^{1}$, Moumen Darcherif ${ }^{1}$, Abdellah El Barkany ${ }^{2}$ \\ ${ }^{1}$ ECAM-EPMI, 13 Boulevard de l'Hautil, 95092 Cergy-Pontoise \\ ${ }^{2}$ Mechanical Engineering Laboratory, Faculty of Sciences and Techniques, Sidi Mohammed Ben Abdellah \\ University, Fez, Morocco
}

\begin{abstract}
The target of this study is to find an appropriate research framework on the basis of the main published works designed to resolve the scheduling issues with energy aspect consideration. Relatively, the major part of work is placed in the context when modeling scheduling is subject only to one constraint and criteria, which in reality is not constantly the case. This study is handled in the framework where job transactions are interrupted at the similar time by set of technological, environmental and especially energetic constraints that has been previously little discussed in the literature. As soon as the objective functions, constraints, models, approaches and the scheduling environments that are defined as the fundamental elements of scheduling problems resolution are determined, a literature review of the present state of the art is introduced. Then, an analysis is carried out to highlight the fundamental ideas leading to fix a future research area that still require a huge deal of attention.
\end{abstract}

\section{Introduction}

The actuality of climate change and the magnitude of this total challenge are well established. The need and urgency for action is now recognized by mutually the scientific researchers and political leaders. Since Copenhagen, a global ambition level has been defined, with the purpose of preventing a warming of more than $2^{\circ} \mathrm{C}$ compared to the pre-industrial era.

Climate change is intrinsically linked to our dependence on fossil resources: it results from the fact that humanity, by burning at high speed fossil organic fuels accumulated over millennia, releases big quantities of carbon into the atmosphere. Climate change and energy scarcity are two front of the same coin; these crises cannot be dealt with separately. To cope with this, it is obviously necessary to spotlight on energy demand in the various activities sectors and mainly, the manufacturing one.

This augment in energy consumption has become a considerable interest in particular for energy-intensive industries, counting mussels, chemicals and petroleum. For example, the energy use of the ferrous metal casting and pressing industry (mainly injection moulds and stamping dies) represents about $28.8 \%$ of the overall energy consumption of the industrial area in 2014.

Under those conditions, minimizing energy consumption represents a major challenge in these environments, leading not only to an efficient industrial system in terms of cost, quality and time. But also towards a global system that effectively responds to the enormous environmental, economic and technological hazards at the same time. This optimization can be attained $\mathrm{d}$ by using several keys representing problems made complex by the application of the real constraints of the environments in question. In particular, the problem of scheduling tasks which is considered as one of the significant tools that have an impact on machine consumption by affecting the quality, efficiency and cost of the production implementation.

In this context, this research work revolves around a literature review and a detailed bibliography analysis of scheduling problems aimed at optimizing mainly energy criteria with energy considerations in order to fix the critical issues that still necessitate a great interest and determine future research area.

The rest of this paper is structured as follows. After explaining the research approach adopted to select interesting documents, in Section 2, fundamentals elements, which are considered essential for the definition of a scheduling problem with energy aspect are introduced in section 3. Section 4 reports a global literature review for the production scheduling problems under investigation. Finally, conclusions are drawn together with perceptions for future research in Section 5.

\section{RESEARCH APPROACH}

Given that, the interesting papers are dispersed on different sources, it is extremely arduous to establish an exhaustive bibliographic study of the studied problem. Consequently, a documentary research tool is used in this research work. It is divided on publication paper, conference papers, student reports and theses which have been deeply examined so as to attain a comprehensive

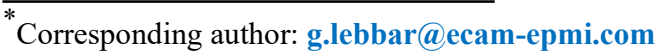


bibliography on the scheduling problem with energetic considerations like: Scopus-Elsevier, Taylor and Francis Group, IEEEXplore, Springer Link...

Firstly, the following keyword was used: 'scheduling' 'energy constraint' and 'energy consumption'. This procedure originally produced interesting papers; afterwards, each paper has been attentively investigated to erase those that are not linked to the requested search criteria. Finally, the pertinent papers are chosen and arranged by year of publication as mentioned in Figure 1.

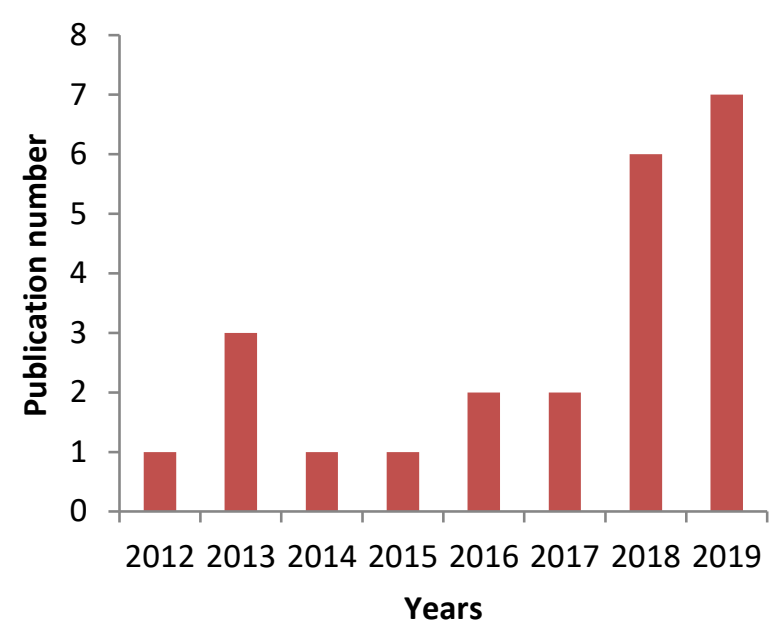

Fig.1: Evolution of publications of scheduling problems with energy constraints

It can be noted that, the growth of publications for the studied problem has increased rapidly in recent years, although during a few years the quantity of publications has been almost invariable, there is a trend of progress in the research for flow shop type of scheduling problems with energetic aspect, which further demonstrates the importance of looking more closely at these types of problems.

\section{Fundamental elements of production scheduling with energetic aspects}

According to [1], Scheduling the operation of an industrial production system consists in managing the allocation of resources over time, while optimizing a set of criteria as much as possible. From an industrial point of view, it consists of a set of $\mathrm{N}$ jobs $\mathrm{J}=(\mathrm{J} 1, \mathrm{~J} 2, \ldots ., \mathrm{Jn})$ that have to be handled on a set of $m$ machines $\mathrm{M}=(\mathrm{M} 1, \mathrm{M} 2, \ldots, \mathrm{Mm})$ in accordance with the order which mainly depends on the type of scheduling workshop studied.

Basing on the references that had handled the scheduling problems with energetic consideration, we selected the most used configurations in the literature.

In our research work, we are mainly concerned with the scheduling problem with energetic aspect, whose main target is to find the best possible scheduling sequence that not only minimizes processing time criteria such as delay, advance, makespan and flow time. But also energy
Consequently, figure 2 includes different the scheduling environments adopted, the developed models, the adopted approaches, the targeted criteria and the constraints considered in the literature.

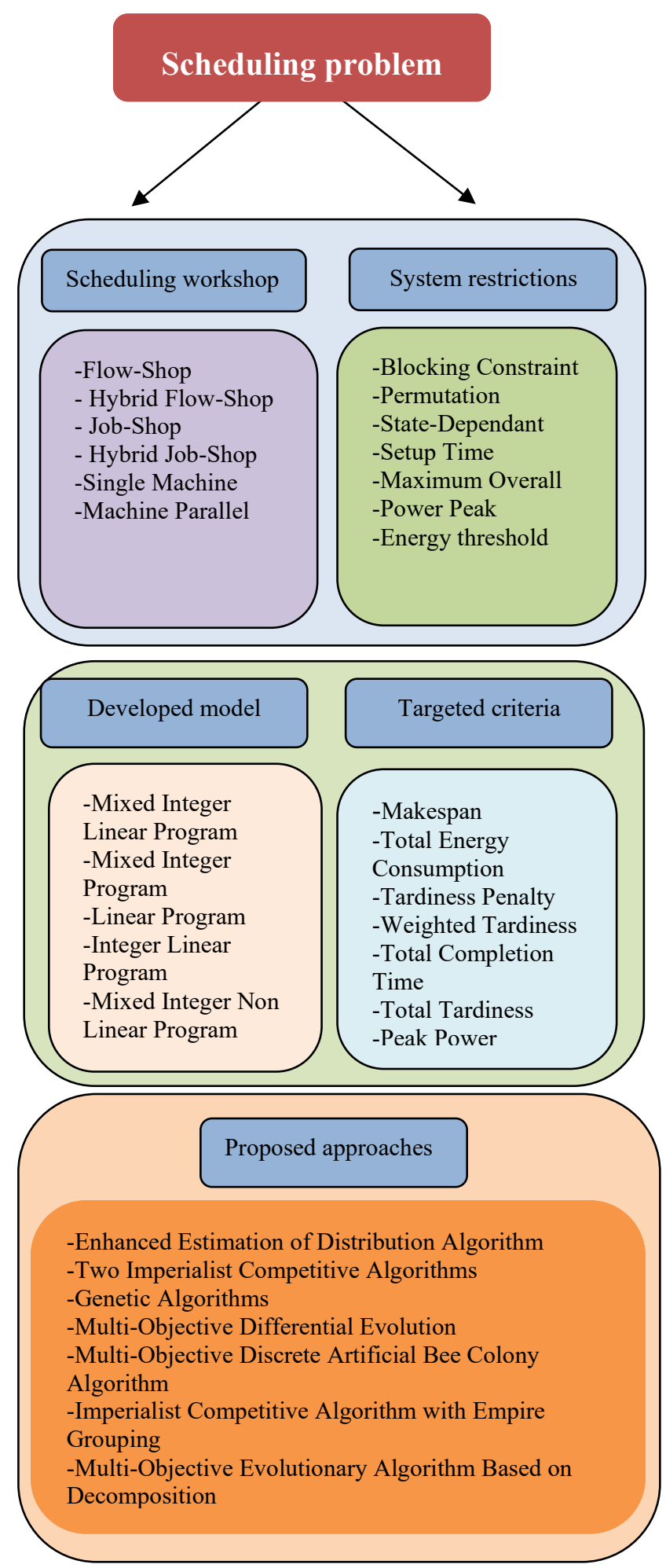

Fig. 2: The different configurations handled in the literature

efficiency measures such as minimizing energy consumption, energy costs, peak power and total carbon emissions. 


\section{Production scheduling with environmental aspects-literature review}

Among the studies that have been published in this direction, Yan et al. [2] handled the hybrid flow shop scheduling problem (HFSP) aiming at minimizing makespan and total energy consumption with a multistage optimization approach. Luo et al. [3] adressed the hybrid flow shop scheduling problem (HFS) where the electric power cost under time-of-use (TOU) electricity prices and production efficiency are considered, to handle this problem, a multi-objective ant colony optimization algorithm was inroduced. An original technique to modify the scheduling of the jobs to account for peak power is suggested by Bruzzone et al. [4]. To optimize maximum of completion time and total energy consumption in flexible flow shop scheduling, a genetic-simulated annealing algorithm have been developed by [5]. Luo et al. [6] introduced a novel ant colony optimization aiming at minimizing the electricity consumption cost for the hybrid flow shop scheduling problem. An improved particle swarm optimization (PSO) have been suggested by [7] for solving the flexible flow shop scheduling problem. Lei et al. [8] developed a lexicographical tool to optimize total tardiness and total energy consumption considered as key target and as non-key one, respectively. A multi-objective optimization scheduling model where cost-weighted processing quality, makespan, and energy consumption, have been optimized, is suggested by Jiang et al. [9], they have applied an improved NSGA-II approach regarding crossover and mutation strategy aiming to surmount the prematurity of population. Mokhtari and Hassni [10] have handled the HFS problem (FJSP) by suggesting a multi-objective optimization model with three objective functions namely, total completion time, the total availability of the system, and total energy cost of production and maintenance operations. Liu et al. [11] have addressed the fuzzy flow shop scheduling problem where the target was to transmit operations to the ressources and to fix the optimal permutation and state shift of each machine so as to minimize tardiness and energy consumption. To handle the problem, they have proposed two common model corresponding implementation and heuristics and then they have combined them with the famous genetic algorithm. A multi-objective optimization algorithm (EAMOA) is proposed by [12] to optimize simoultanously the makespan and the energy consumptions for a HFS problem with the consideration of the setup energy consumptions. A Multi-criteria MILP formulation for solving the HFS scheduling is developed by [13]. In another study [14], a MILP formulation is suggested to look for the optimal solutions for the HFS Scheduling Problem. The purpose of this study was to optimize the total production costs counting transportation and energy costs, basing on a (GA) with a detailed matrix encoding technique. Salido et al. [15] have handled a jobshop scheduling problem designed to optimize energy efficiency, robustness, and makespan. Wang et al [16] have investigated the flow shop scheduling with the constraint of the limited buffer between consecutive resources and the aim of minimizing maximum completion time and energy consumption. To resolve this problem, they have proposed a multi-objective parallel variable neighborhood search (MPVNS) algorithm. Chengzhi Guo and Deming Lei [17] suggested a novel imperialist competitive algorithm (ICA) for solving the multi-objectif FJSS problem. The main target of their work was to optimize total tardiness and makespan with a threshold of the total energy consumption imposed. Zhong et al [18] have solved the multi-objective based green flow shop scheduling problem (MOPFS) using a hybrid shuffle frog leaping algorithm based on the cuckoo search algorithm (HFLCS), the performance measures was the makespan and energy consumption. Recently, Lu et al. [19] have developed a MIP model for solving the HFS scheduling problem considering energy consumption and noise pollution. Then, to handle this problem, they suggested a MOCGWO approach with the aim to balance exploration and exploitation by integrating the qualities of variable neighborhood search (VNS) for intensification and cellular automata (CA) for diversification. Min et al. [20] addressed the FJSS shop scheduling problem with transportation constraints, the aim of their study is to minimize energy consumption and makespan. Li et al [21] investigated the Energy-efficient hybrid flow shop scheduling problem with makespan ,total tardiness, and total energy consumption criteria. The problem resolution was based on a new Pareto dominance and a two-level imperialist competitive algorithm (TICA). Zhang et al. [22] proposed a scheduling approach for the MOFJSP and the resultant mathematical model, considering both the total production energy consumption and makespan. Besides, transportation with its influance on the energy consumption was considered in their model for realistic application. An approached tool based on the NSGA-II was applied to solve the considered problem. Zhou et al. [23] have solved the HFS scheduling problem with fuzzy processing time by proposing a multi-objective formulation that minimizes simultaneously total energy consumption andtotal weighted delivery penalty. In their study they have considered Setup times as sequencedependent and in-stage parallel, resources are unrelated. Jiang et al. A MILP model have been suggested by [24] for solving the HFS scheduling problem with restricted buffers. The aim of their study was to minimize nonprocessing energy (NPE) and the total weighted tardiness (TWT).

\section{Analysis and discussion}

The greater part of the research that has been published has paying attention on the optimization of energy consumption in conjunction with makespan in order to realize a production environment that uses maximum resources but with minimal energetic consumption. For manufacturing environments, the HFS is the most studied. With consider to resolution approaches the majority of research teams have leaned towards approximate resolution methods. Finally, it can be noted that a lot of energetic and technological restrictions related to the short planning energetic systems has been handeled in the literature. 


\section{Conclusion}

In the recent years, with the massive increase in energy consumption and its dangerous consequences essentially affecting the international problem of climate change. industries are seeking to improve their production processes by paying particular attention to the energy aspect. In this context, the research on the integration of the problems encountered at the level of the industrial environment by considering the energy constraints with a very important weight has been of great interest in the last decades. Among those problems, short-term production planning - or scheduling is considered as one of the key instruments that make it possible to achieve an economically and energetic optimal production system. As a result, studies resolving the scheduling problem environmental aspect consider actually the energetic restriction as a perturbation to the production process. In this context, our research work focuses on a comprehensive literature review of the works that have addressed, a and analyzed workshop scheduling issues with energy considerations. The analysis of the bibliography can led to fix research perspectives that still necessitate attention.

In perspective, we intend to develop a mathematical formulation for the scheduling environment having an important historical place in the literature by considering mainly the energetic criteria and the energetic and technological constraints simultaneously. Regarding solution approach, a hybrid evolutionary algorithm will be considered since this combination will be adjusted by a strong theoretical complexity.

\section{References}

[1]. F.A. Rodammer et K. Preston White, A recent survey of production scheduling, IEEE Transaction on Systems, Man and Cybernetics, 618, (1999).

[2]. J. Yan, L. Li, F. Zhao, F. Zhang, Q. Zhao, A multilevel optimization approach for energy-efficient flexible flow shop scheduling, J. Cleaner Prod. 137, 1543-1552, (2016).

[3]. H. Luo, B. Du, G.Q. Huang, H. Chen, X. Li, Hybrid flow shop scheduling considering machine electricity consumption cost, Int. J. Prod. Econ. 146, 423-439, (2013).

[4]. A.A.G. Bruzzone, D. Anghinolfi, M. Paolucci, F. Tonelli, Energy-aware scheduling for improving manufacturing process sustainability: a mathematical model for flexible flow shops, CIRP Ann. - Manuf. Technol. 61, 459-462, (2012).

[5]. M. Dai, D.B. Tang, A. Giret, M.A. Salido, W.D. Li, Energy-efficient scheduling for a flexible flow shop using an improved genetic-simulated annealing algorithm, Robo. Comput.-Int. Manuf. 29 (5),418-429, (2013).

[6]. H. Luo, D. Du, G.Q. Huang, H.P. Chen, X.L. Li, Hybrid flow shop scheduling considering machine electricity consumption cost, Int. J. Prod. Econ. 146 (2), 423-439, (2013).

[7]. D.B. Tang, M. Dai, M.A. Salido, A. Giret, Energy-efficient dynamic scheduling for a flexible flow shop using an improved particle swarm optimization, Comput. Ind. (30) 223-232, (2015).

[8]. D.M. Lei, L. Gao, Y.L. Zheng, A novel teachinglearning-based optimization algorithm for energy-efficient scheduling in hybrid flow shop, IEEE Trans. Eng. Manag. 65 (2) 330-340, (2018).

[9]. Jiang, Z., \& Le, Z. Study on multi-objective flexible job-shop scheduling problem considering energy consumption. Journal of Industrial Engineering and Management (JIEM), 7(3), 589-604, (2014).

[10]. Mokhtari, H., \& Hasani, A. An energy-efficient multi-objective optimization for flexible jobshop scheduling problem. Computers \& Chemical Engineering, 104, 339-352, (2017).

[11]. Liu, G. S., Zhou, Y., \& Yang, H. D. Minimizing energy consumption and tardiness penalty for fuzzy flow shop scheduling with state-dependent setup time. Journal of cleaner production, 147, 470-484, (2017).

[12]. Li, J. Q., Sang, H. Y., Han, Y. Y., Wang, C. G., \& Gao, K. Z. Efficient multi-objective optimization algorithm for hybrid flow shop scheduling problems with setup energy consumptions. Journal of Cleaner Production, 181, 584-598, (2018).

[13]. Schulz, S. A multi-criteria MILP formulation for energy aware hybrid flow shop scheduling. In Operations Research Proceedings 2016 (pp. 543-549). Springer, Cham, (2018).

[14]. Schulz, S., Neufeld, J. S., \& Buscher, U. A multiobjective iterated local search algorithm for comprehensive energy-aware hybrid flow shop scheduling. Journal of Cleaner Production, 224, 421-434, (2019).

[15]. Salido, M. A., Escamilla, J., Barber, F., Giret, A., Tang, D., \& Dai, M. Energy efficiency, robustness, and makespan optimality in job-shop scheduling problems. AI EDAM, 30(3), 300312, (2016).

[16]. Wang, F., Deng, G., Jiang, T., \& Zhang, S. Multi-objective parallel variable neighborhood search for energy consumption scheduling in blocking flow shops. IEEE Access, 6, 6868668700, (2018).

[17]. Guo, C., \& Lei, D. Multi-objective Flexible Job Shop Scheduling Problem with Energy Consumption Constraint Using Imperialist Competitive Algorithm. In International Conference on Intelligent Computing (pp. 659669). Springer, Cham, (2018).

[18]. Zhong, L. C., Qian, B., Hu, R., \& Zhang, C. S. The Hybrid Shuffle Frog Leaping Algorithm Based on Cuckoo Search for Flow shop 
Scheduling with the Consideration of Energy Consumption. In International Conference on Intelligent Computing (pp. 649-658). Springer, Cham, (2018).

[19]. Lu, C., Gao, L., Pan, Q., Li, X., \& Zheng, J. A multi-objective cellular grey wolf optimizer for hybrid flow shop scheduling problem considering noise pollution. Applied Soft Computing, 75, 728-749, (2019).

[20]. Dai, M., Tang, D., Giret, A., \& Salido, M. A. Multi-objective optimization for energy-efficient flexible job shop scheduling problem with transportation constraints. Robotics and Computer-Integrated Manufacturing, 59, 143157, (2019).

[21]. Li, M., Lei, D., \& Cai, J. Two-level imperialist competitive algorithm for energy-efficient hybrid flow shop scheduling problem with relative importance of objectives. Swarm and Evolutionary Computation, (2019).

[22]. Zhang, B., Pan, Q. K., Gao, L., Li, X. Y., Meng, L. L., \& Peng, K. K. A multiobjective evolutionary algorithm based on decomposition for hybrid flow shop green scheduling problem. Computers \& Industrial Engineering, 136, 325-344, (2019)..

[23]. Zhou, B., \& Liu, W. Energy-efficient multiobjective scheduling algorithm for hybrid flow shop with fuzzy processing time. Proceedings of the Institution of Mechanical Engineers, Part I: Journal of Systems and Control Engineering, 0959651819827705, (2019).

[24]. Jiang, S. L., \& Zhang, L. Energy-Oriented Scheduling for Hybrid Flow shop With Limited Buffers Through Efficient Multi-Objective Optimization. IEEE Access, 7, 34477-34487, (2019). 